\title{
Aproximación al proceso de reinserción de jóvenes que han vivido un proceso carcelario en los Centros de Internamiento para Adolescentes de Hidalgo ${ }^{1}$
}

\author{
A Study on the Process or Reinstallation into Society of Youngsters Who Have Been in Jail in \\ Centros de Internamiento para Adolescentes in Hidalgo State, Mexico \\ María Elena Pérez Maldonado ${ }^{a}$, Juan Antonio Taguenca Belmonte ${ }^{b}$
}

\begin{abstract}
:
Advances of a $\mathrm{PhD}$ research that aims to analyze the process of reintegration of young people, who have lived a prison process in the Detention Centers for Adolescents. We studied the subjectivity of the adolescent and the sociocultural factors that intervene in the decisions that the young person takes when he reinserts himself in his environment and tries to resume his daily life. Methodological tools from ethnography are used: life stories, observation and exhaustive interviews.
\end{abstract}

Keywords:

Young People. Paths. Deviated Behavior. Reintegration.

\section{Resumen:}

Se presentan avances de una investigación de doctorado que tiene como objetivo analizar el proceso de reinserción de jóvenes que han vivido un proceso carcelario en los Centros de Internamiento para Adolescentes. Para ello estudiamos la subjetividad del adolescente y los factores socioculturales que intervienen en las decisiones que el joven toma cuando se reinserta a su entorno e intenta retomar su vida cotidiana. Se utilizan herramientas metodológicas procedentes de la etnografía: relatos de vida, observación y entrevistas a profundidad.

\section{Palabras Clave:}

Jóvenes. Trayectorias. Conductas desviadas. Reinserción

Recibido: 31 de julio de 2018

Dictaminado: 20 de agosto de 2018

Segunda versión: 30 de agosto de 2018

Aceptado: 3 de octubre de 2018

${ }^{1}$ El texto fue presentado como ponencia en XXXI Congreso Internacional de la Asociación Latinoamericana de Sociología, Montevideo 2-8 de diciembre de 2017.

\footnotetext{
a Estudiante de doctorado de Ciencias Sociales, Instituto de Ciencias Sociales y Humanidades, Universidad Autónoma del Estado de Hidalgo, País: México·Email: lna_88pm@hotmail.com

b Profesor Investigador del Área de Ciencias Políticas y Administración Pública, Instituto de Ciencias Sociales, Universidad Autónoma del 


\section{Introducción}

En el presente reporte, introducimos algunos avances de la investigación que está llevando a cabo una alumna del programa de Doctorado en Ciencias Sociales. La investigación tiene como objetivo el análisis de la trayectoria de vida de los jóvenes que han infringido la ley y que han ingresado en una prisión para menores (Centros de Internamiento para Adolescentes). Se trata de identificar aquellos momentos importantes en la vida de los jóvenes, hasta cierto punto determinantes en los procesos de socialización, que los llevaron a realizar diversas prácticas consideradas desviadas y, por ende, a consolidar una carrera delictiva.

Las conductas denominadas desviadas o delictivas (considerando los valores centrales de la sociedad o las normas y leyes que garantizan su reproducción), pueden ser analizadas desde diferentes disciplinas y enfoques, como son la sociología jurídica, la psicología, la antropología y la sociología de la desviación. Esta última será el enfoque teórico utilizado para analizar, como decíamos, el problema de la trayectoria desviada del joven transgresor. La conducta delictiva es criminalizada y se trata de castigar al infractor de la ley. Los procesos de criminalización se relacionan con formas de control social a partir de etiquetas negativas impuestas a aquellas personas que se consideran atentan en contra del orden y la reproducción social. No obstante, recordemos, la desviación social es una perspectiva que se construye desde el poder del Estado y de aquellos grupos de presión que dictan cierta moralidad colectiva. La llamada teoría de las subculturas surge a contra corriente de esta postura legal, que no reconoce el surgimiento del sentido de ciertas prácticas grupales y sociales a partir de determinados contextos.

Downes \& Rock (2011) consideran que la definición de la desviación social es tan ambigua que no podemos conformarnos con una sola explicación; la definición, sin duda, dependerá del contexto en el que se presenten determinadas actividades o prácticas validadas por los propios individuos y grupos. Cada situación se desarrolla dentro de cierto contexto cultural, económico, político, etcétera, en dónde se realizan infinidad de intercambios que definen la naturaleza de las relaciones sociales y el tipo de vínculos afectivos que les acompañan. De ahí que sea interesante investigar la importancia de las bandas, las biografías o trayectorias sociales, el lenguaje y los objetos que se intercambian en determinadas situaciones, con la finalidad de conocer el sentido de las conductas o de la acción social. La desviación se define a partir de la construcción de las normas morales generales de una sociedad específica, sin embargo, cada vez más se reconoce que las sociedades son heterogéneas y que en ellas se da una lucha en torno a lo que se considera moralmente correcto.
Es así que el objetivo de esta investigación es analizar los momentos que para los jóvenes que han infringido la ley son importantes en la conformación de su trayectoria desviada. En una palabra, deconstruir las situaciones e identificar aquellos aspectos socioculturales que enmarcaron las conductas desviadas y delictivas de los jóvenes.

\section{Marco teórico conceptual}

Para esta investigación utilizaremos los planteamientos del interaccionismo simbólico de autores como: Blumer (1981), Goffman (2007) y Becker (2009), con el objetivo de explicar este proceso de deconstrucción de una carrera delictiva. La pregunta básica es cómo se llega a tener una conducta desviada, pensando que la misma no nace de la noche a la mañana, sino que es resultado de infinidad de intercambios y de experiencias que se transmiten y que dan como resultado un conocimiento práctico que se incorpora como hábito al sujeto. El criminólogo Edwin Sutherland (1983) denomina a este proceso "socialización por impregnación".

Con base en la teoría de Howard (2009), aquel que es definido como delincuente porta una etiqueta social degradante. En este sentido, determinadas instituciones de salud o correccionales pueden provocar este efecto etiquetador en sus internos, más allá de que no sea este su objetivo explícito. Para nuestro caso, los jóvenes que entrevistamos, como parte de esta investigación son delincuentes (con toda una serie de equivalencias simbólicas. como son: vagos, mal vivientes, peligrosos, adictos y violentos, entre otras). Esto por el hecho de haber ingresado a la institución penitenciaria, lo que socialmente los etiqueta como delincuentes.

Como decíamos más arriba, la desviación no sólo es un problema jurídico sino también moral, sociológico y político. La misma es consecuencia de los procesos de intercambio, es decir, del contacto continuo entre las personas, lo que se analiza con el término de "socialidad". La desviación social emerge entonces de la reacción social que provoca determinado comportamiento que lesiona a la colectividad por ser considerado incorrecto o desviado. Por eso el proceso de rotulación o etiquetamiento no sólo es un atributo impuesto desde la ley, sino también un proceso derivado de las relaciones sociales que resultan en procesos de diferenciación y distinción entre las personas.

Los grupos sociales crean la desviación, al establecer las normas cuya infracción constituye una desviación y al aplicar esas normas a personas en particular y etiquetarlas como marginales. [...] La desviación [...] es una consecuencia de la aplicación de reglas y sanciones sobre el "infractor" a manos de terceros (Becker, 2009: 28). 
La teoría de Becker (2009) explica porque algunos de los jóvenes que ingresaron como primo-delincuentes a prisión al salir están predispuestos a realizar una carrera delictiva, asumiendo de esta manera la etiqueta de delincuente que la sociedad les dio al entrar en prisión. También puede suceder que la familia o el barrio jueguen un papel importante en la conducta transgresora y el ingreso a una institución correccional del Estado -con su consecuente estigmatización- sea tan sólo una parte más de la trayectoria delincuencial.

En este reporte preliminar de investigación se trata de analizar la carrera delictiva de los jóvenes en cuanto sus causas. Uno de los aspectos a tratar son los procesos de socialización que conducen hacia determinadas conductas delictivas. La teoría de Berger y Luckmann desarrolla el tema sobre la socialización primaria y secundaria. La misma nos sirve para llevar a cabo los análisis de algunos casos que han incorporado determinadas conductas consideradas desviadas desde la infancia con la familia, o con los grupos de referencia, amigos de la escuela y, en otros casos, los aprendidos en el interior de la prisión (Berger \& Luckmann, 2015).

El individuo, según Berger y Luckmann (2015), no nace integrado como miembro de una sociedad, antes bien se convierte en miembro de ella a partir de las relaciones con los otros. La socialización primaria es quizás la más importante en el proceso de vida de un individuo, ya que es donde recibe sus primeros aprendizajes de la familia o las personas cercanas que le brindan cuidado durante la infancia; es único y será la base para las diversas socializaciones secundarias que viva después a través de las instituciones: escolar, laboral, religiosa o política. Recordemos que la socialización consiste en internalizar patrones de conocimiento, tradiciones, valores, afectos de forma subjetiva, es decir, a manera de experiencias de vida de acuerdo a las reglas morales de la sociedad para ser reproducidas como integrante de un grupo 0 institución.

Becker (2009) también plantea que los sujetos que cometen actos delictivos constantemente respetan sus propias normas o valores llegando a naturalizarlos, los viven como parte normal de su cotidianeidad. En este mismo sentido, Matza (2014) estudia cómo los sujetos justifican las prácticas transgresivas a través de ciertos mecanismos expresados por medio del lenguaje, que denomina: "técnicas de neutralización". Lo anterior permite comprender que los jóvenes que han quebrantado la ley no son parte de una contracultura, sino que son sujetos que están conscientes de que sus actos van en contra de ciertas normas morales, pero que han aprendido a adaptar sus prácticas en una visión que niega o neutraliza el daño provocado al otro mediante el delito cometido.
Respecto al internamiento institucional, sin duda Goffman es uno de los sociólogos más importantes para comprender la dinámica del encierro forzado, los efectos que provoca en los internos y los mecanismos de supervivencia que éstos adoptan para enfrentar la situación. El concepto "de institución total", acuñado por el sociólogo canadiense, habla de esta tendencia absorbente que moldea a los internos que tienen que convivir obligadamente en diversas actividades y prácticas, incluyendo las más íntimas.

Dentro de las prisiones, como en muchas otras instituciones, se establecen relaciones de poder entre la población internada. Estas relaciones son complejas y devienen de las colusiones entre grupos, de las relaciones entre el mundo del personal y de los internos, la corrupción y los recursos financieros, entre otros. Intervienen actores y situaciones diversas que pueden estudiarse a través de los testimonios, como parte de los procesos de socialización y aprendizaje informal que se adquiere al interior de estas instituciones.

La perspectiva de Goffman subraya los efectos que provoca el internamiento forzado sobre los sujetos. Cuando el interno ingresa, cambia su posición social y su identidad. Es despojado de ciertas características que conformaban, su "yo" interno y que lo hacía parte de una sociedad o grupo determinado. A través de una serie de rituales degradantes, humillaciones, provocaciones al yo, pérdida de la economía personal y exhibición contaminadora, el sujeto es desbaratado simbólicamente por medio de una serie de procesos de mortificación (Goffman, 2007:27). Por un lado, tenemos una serie de rituales de degradación y, por el otro, las formas de adaptación o subsecuencia con la que los internos enfrentan la situación: colonización, rebeldía e infantilización.

En una visión más extrema, se dice que las prisiones son fábricas de criminales, resultan lo contrario de aquello que pretenden: la reinserción social. Lo que es un hecho, es que la prisión, como cualquier otra institución, establece una serie de prácticas que socializan los sujetos mediante la interacción física y simbólica. Algunas de ellas son formales y forman parte de los programas institucionales, pero muchas otras son informales y se relacionan con valores provenientes de los lugares de ocio de los barrios y de las bandas juveniles. La internalización es un proceso en el que el individuo significa los acontecimientos de la realidad objetiva, pero que interpreta de manera subjetiva, de acuerdo a sus experiencias biográficas; dicho proceso sirve en todo momento para la comprensión de la realidad subjetiva en la que viven los reclusos y para la constante aprehensión del mundo objetivo en que se desarrolla el encierro en la cárcel, y está sujeto a un proceso de socialización. 
La reincidencia, como parte del proceso de reinserción, puede ser explicada también a través de Becker, quien considera que cuando un individuo entra en contacto con un grupo desviado institucionalizado, como la cárcel, encuentra a sus pares y aprende a llevar a cabo su actividad delictiva con mejor destreza, aprendiendo a realizar los actos delictivos que realizaba y otros con mayor eficiencia y eficacia.

De esta manera, al ingresar en un grupo desviado organizado o institucionalizado, es más probable que el individuo continúe por el camino de su desviación. Por un lado, ha aprendido cómo evitarse problemas, y por el otro, ha incorporado una lógica que le permite continuar sin reprochárselo (Becker, 2009: 57).

Con el concepto de "deriva", de Matza, se puede explicar que el tener una carrera delictiva no determina que sea la única actividad a la que se dedican los jóvenes, o que tengan una estructura de valores conforme a la conducta delictiva como una subcultura, pues pueden alternar actividades legales con las ilegales de forma simultánea: "La deriva es una fase en la cual los jóvenes pueden quebrantar ciertas leyes en determinadas ocasiones y contextos pero sin estar, de modo alguno, condicionados a hacerlo" (Matza, 2014:14).

Algunos jóvenes pueden vivir exclusivamente de la actividad delictiva, pero algunos otros pueden tener un trabajo legal, ir a la escuela, tener actividades sociales y deportivas, y convivir de acuerdo a las normas morales establecidas socialmente, pero en situaciones específicas están dispuestos a quebrantar la ley. El delincuente juvenil sostiene alguna relación con el orden legal y con sus exigencias, principios y doctrinas (Matza, 2014:39).

Por otro lado, la condición juvenil va a influir en las configuraciones subjetivas que el sujeto hace sobre su vida y su conducta, por lo que en esta investigación es de gran relevancia considerar y definir a la juventud como una categoría de análisis, no sólo como referente etario.

Los jóvenes, en esta sociedad moderna, se han convertido en uno de los grupos de mayor análisis científico, debido al impacto que han tenido como grupo cultural en diferentes situaciones y problemáticas; por ejemplo: movimientos sociales, drogas, violencia, educación, actividades ilegales, deportes, pandillas, política, cárceles, etc.

Dentro de las ciencias sociales, la condición juvenil se refiere al imaginario social institucionalizado, que muestra el "deber ser" joven, es decir, existe una configuración institucionalizada de cómo debe ser y comportarse un sujeto al que se le considera joven.

Los jóvenes tienen una determinada forma de actuar, vestimenta específica de acuerdo al sector juvenil al que pertenezca, consumo de ciertos productos que lo van a identificar como tal, etc. Los jóvenes tienen prácticas y formas de expresión, normas de comportamiento propias y una manera especial de relacionarse con su entorno social (Reguillo, 2010).

Kessler (2004), Saraví (2015), Azaola (1990); Valenzuela Arce, Nateras y Reguillo (2007), son algunos de los autores que han dado cuenta de la relación entre juventud y violencia o conductas delictivas. Estas investigaciones nos muestran un panorama del contexto socio-cultural latinoamericano al que se enfrentan algunos de los estratos de la población juvenil.

Algunos autores rechazan el tiempo cronológico como base del concepto de juventud, como Taguenca (2016), por lo que se plantea el ser joven como un tiempo vivencial según los estratos generacionales y sociales en los que está ubicado. De este modo, la experiencia de los jóvenes viene enmarcada por la posición social a partir de un proceso histórico determinado, por lo que proponen entender las variaciones en el tiempo de las formas de producción de los sujetos como una "estratificación de las experiencias".

En este sentido, es importante tomar en cuenta el contexto sociocultural en el que viven los sujetos de estudio, ya que será un factor que delimite el camino por el cual seguirán los jóvenes, ya sea la reincidencia o una nueva forma de vida conforme a la ley.

Para el análisis del contexto en el que se encuentran inmersos los sujetos de estudio, planteamos un panorama general sobre el contexto latinoamericano moderno que diversos autores han llamado como "modernidad tardía", "modernidad líquida", con respecto a la definición y significación de ciertas conductas como delictivas y a las nuevas reformas a la ley que tienen impacto social.

Investigaciones como las de Garland (2012) y Wacquant (2012), entre otros, se ocupan del contexto sociocultural de "modernidad tardía" o neoliberalismo, que a partir de las múltiples transformaciones culturales y estructurales da como resultado cambios y reformas a las leyes que rigen una nación.

Garland (2012) considera que la política de criminalidad y los mecanismos de control del delito se alinearon con la cultura y las relaciones sociales contemporáneas. Los nuevos dispositivos de control del delito están dirigidos por las políticas de libre mercado y la forma de organización social distintiva de la modernidad tardía.

De acuerdo con Saraví (2015), el contexto neoliberal ha dado como resultado una fragmentación social, la cual se refiere a la exclusión de los sectores sociales más vulnerables como: jóvenes, indígenas, mujeres, migrantes y desempleados. Esta situación de exclusión hace que, en algunas ocasiones, los jóvenes se vean orillados a tener conductas desviadas como vía de acceso a la estructura social "normal". 
A pesar de que en la ley se estipula que la prisión es la última medida que se debe tomar como forma de sanción de conductas, en la práctica esto es diferente. Según Wacquant (2010:99), existe un encarcelamiento desmedido de ciertos sectores de la población que están en una situación económica y social de desventaja. El autor considera que el Estado Penal se ha convertido en una política de criminalización de la miseria, que complementa perfectamente a la economía neoliberal del trabajo asalariado precario y a los nuevos programas sociales que tienen la finalidad de controlar a ciertos sectores de la población, como es el caso de los jóvenes.

\section{Metodología}

En la investigación que se está desarrollando, utilizamos técnicas etnográficas, pues nuestro objetivo es analizar las trayectorias de vida de los jóvenes que han infringido la ley y que en algunos casos han tenido una carrera delictiva. Se pretende comprender la realidad a través de herramientas descriptivas como: la observación, las entrevistas en profundidad y los relatos de vida.

La frase metodología cualitativa se refiere en su más amplio sentido a la investigación que produce datos descriptivos, las propias palabras de las personas, habladas o escritas, y la conducta observable (Taylor \& Bogdan, 1994:20).

Según Taylor \& Bogdan (1994), el investigador debe tomar en cuenta el contexto histórico y el contexto en el que se encuentra el objeto de estudio, nada debe darse por entendido, el investigador debe mirar por sobre sus propias creencias o prejuicios, viendo todo como algo nuevo y valioso.

Para el caso específico de esta investigación, debido a que las conductas desviadas, delictivas son en su gran mayoría, secretas y ocultas, la información es restringida. Es muy difícil tener acceso a los informantes y el investigador generalmente tiene que realizar un considerable esfuerzo para obtener la confianza de ellos, tal como lo mencionan autores que se ocupan de este estudio temático:

Quienes estudian a los delincuentes juveniles muy rara vez son admitidos en los hogares de estos, o en sus escuelas 0 en sus ámbitos más privados (Downes \& Rock, 2011:51).

De esta manera, para facilitar el trabajo de investigación, se contactó a los sujetos de estudio a través de la prisión especializada para menores, conocida como Centro de Internamiento para Adolescentes de la ciudad de Pachuca, impartiendo un taller de cuento; de la misma manera se solicitará el apoyo del personal de la institución que tenga contacto con algún joven que ya haya salido de prisión. Como una primera aproximación, se realizó observación de la experiencia de los jóvenes que se encuentran dentro del Centro de Internamiento para Adolescentes de la ciudad de Pachuca.

Una segunda técnica a utilizar es la de relatos de vida, la misma nos sirvió para comprender e identificar cuáles fueron los momentos importantes para los sujetos con respecto a la conducta delictiva, es decir, las situaciones clave que definieron su llegada a la institución y en algunos casos su carrera delictiva.

Se entrevistaron a mujeres y hombres jóvenes, que han tenido por lo menos una experiencia de encierro en un Centro de Internamiento para Adolescentes, tratando de ubicar los momentos que fueron decisivos en su trayectoria, mismos que dieron como consecuencia su llegada a la institución. De la misma manera, se indagó sobre sus planes futuros y si en éstos está continuar con su trayectoria delictiva o tomar un camino diferente y, por tanto, mantenerse de acuerdo a las normas morales y legales establecidas en la sociedad.

\section{Análisis y discusión de datos}

¿Cómo se llega a la conducta delictiva? Ya sea que haya sido un acto espontáneo o una acción planificada y estratégicamente bien organizada, la culminación de un acto delictivo debe tener un precedente, algún detonante o cúmulo de situaciones que llevaron al joven a cometer una conducta ilícita.

La conducta delictiva es parte de lo que en sociología se conoce como conductas desviadas. Como se mencionó en apartados anteriores, las conductas desviadas según Becker (2009) son aquellas que han violado alguna norma establecida socialmente dentro de un contexto determinado, que en palabras del autor, "la desviación no es una cualidad del acto que la persona comete, sino una consecuencia de la aplicación de reglas y sanciones sobre el "infractor" (Becker, 2009:28).

Así, la conducta desviada es una respuesta de los otros a las acciones de una persona, es decir, el sujeto es etiquetado por su sociedad como desviado a partir de definir su conducta como algo que va en contra de las normas establecidas. Existen muchos tipos de conductas desviadas y sólo las conductas delictivas o ilegales sancionadas penalmente son la que se tomarán en cuenta para esta investigación. Es decir, aquella que cometen algunos jóvenes y que en algunos casos se vuelve para ellos una forma de vida.

Para algunos adolescentes, la conducta ilícita ha venido siendo un modus vivendi. Desde su infancia, sus padres y familiares toman la conducta ilegal como su forma de trabajo para la manutención familiar. Existen también los casos de adolescentes que vivieron en un entorno familiar que respetaba la ley, pero que en el camino se

\footnotetext{
"De acuerdo con Taylor \& Bogdan (1994) definimos la observación como la interacción social del investigador con los informantes.
} 
encontraron amigos, conocidos o compañeros de escuela con los que iniciaron su trayectoria delictiva, o simplemente se encontraron en una circunstancia que facilitó la decisión para violentar la ley.

La familia es una institución con una estructura de relaciones complejas, que se encarga de brindar a los sujetos los primeros acercamientos con la ley; es decir, transmite, además del soporte emocional, las reglas y normas de comportamiento que le permitirán relacionarse con su contexto social de manera ordenada. En la familia es donde se adquiere el aprendizaje social básico, y los primeros años de vida son para el individuo los momentos más importantes de internalización ${ }^{*}$ de las formas de comportamiento que le dan sentido y objetivan su mundo, pues como dicen Berger y Luckmann (2015), la internalización constituye la base, primero, para la comprensión de los propios semejantes y, segundo, para la aprehensión del mundo en cuanto a realidad significativa y social.

De esta manera, para un niño puede resultar cotidiano algún tipo de violencia o tomar las cosas de los demás sin permiso, pues su grupo socializador inmediato, que resulta ser su núcleo familiar, se le ha transmitido, a través de actitudes de permisividad de dichos actos, que su conducta es correcta o bien, si no es correcta, tiene justificación o se identifica como "normalizada".

Existen casos en los que no sólo son actitudes de permisividad, sino que existe la transmisión de los actos desviados, a través del ejemplo de los padres o de las personas que estén a cargo de la socialización primaria de los niños. Sin embargo, la realidad cotidiana es mucho más compleja que sólo aprehender pautas de comportamiento y realizarlas. El individuo va más allá de ser un receptor de normas y ejecutarlas, como lo dicen algunas teorías positivistas en las que consideran que sólo hay dos opciones de estar en la sociedad: cumpliendo con las normas o violándolas.

De la misma manera, se identifican diversos factores que explican la conducta delictiva juvenil, en ocasiones los jóvenes llegan a cometer actos delictivos sin tener un antecedente familiar, en el que hayan socializado los patrones de conducta, por el contrario, existen casos en los que los jóvenes que delinquieron tuvieron una educación de acuerdo a las normas establecidas y recibieron además afecto y cariño por parte de su familia, como lo dice Rosa, una joven de 18 años de edad que estuvo en el Centro de Internamiento para Adolescentes acusada delincuencia organizada y daños contra la salud:

Cuando vivía en México, mis primos se robaban cosas de la tienda, yo sólo los veía, pero una vez lo

[Es] la aprehensión o interpretación inmediata de un acontecimiento objetivo en cuanto expresa significado, o sea, en cuanto es una manifestación de los procesos subjetivos de otro, en consecuencia se vuelven subjetivamente significativos para mí. quise hacer y mi papá me cachó... me pegó muy feo para que se me quitara la maña... (Testimonio de reclusa).

Tomando como base a Matza (2014), los jóvenes tienen conocimiento sobre las normas de comportamiento establecidas socialmente y pueden vivir de acuerdo a ellas, pero en algún momento se encuentran con alguna circunstancia que les facilita la acción delictiva y la realizan. Para ello, desarrollan técnicas de neutralización como forma de negación de la conducta o minimización del daño. Ejemplo de esto es el caso de Samuel, quien neutraliza su conducta por el hecho de haber recibido violencia en su infancia, quien menciona: "Yo no me arrepiento [de] matar, porque cuando era niño me provocaban y me hacían bullying, pero después fui creciendo..."

Otro ejemplo de cómo los jóvenes ocupan las técnicas de neutralización es el de Santiago. ${ }^{\dagger}$ En algunas pláticas él ha mencionado que no se arrepiente del delito que cometió, pues lo que hizo fue por necesidad, para mantener a su familia. Además, dice que les quitó a personas que tienen mucho dinero, lo cual para él de alguna manera justifica su conducta delictiva, expresando lo siguiente:

Pues yo sentí mucha rabia, cuando sabía que mi mamá no tenía dinero para darle de comer a mi hermano, era cuando me daban ganas de salir a hacer maldad, por así decirlo. Salía a robar, asaltar o cosas peores, pero lo hacía sin pensar. La rabia, furia y droga me cegaban. Me ahogaba la furia y era cuando hacía cosas sin pensar. Pero de todo lo que he hecho no tengo arrepentimiento alguno, sé que lo hice por necesidad y a personas que tenían mucho más que yo y nada les costó prestarme un poco, jefe. ${ }^{\ddagger}$

Como se puede observar, la mayoría de los jóvenes entrevistados tuvieron una socialización primaria, por parte de sus familias, con base en las normas de comportamiento socialmente reconocidas y establecidas en la sociedad, por lo que hace falta indagar sobre la internalización de pautas de comportamiento delictivo fuera del entorno familiar.

Mientras que la familia es la institución socializadora primaria por excelencia, la escuela, los amigos, la pandilla, la iglesia, los medios de comunicación son los grupos donde los individuos llevan a cabo su socialización secundaria. De hecho, de acuerdo con

\footnotetext{
† Es un adolescente de 17 años, que se encuentra recluido en el Centro de Internamiento para Adolescentes de la ciudad de Pachuca.

₹ El fragmento refiere a un escrito elaborado en una sesión del taller de Cuento, impartido por la Maestra María Elena Pérez Maldonado, como parte del trabajo de campo de su tesis de doctorado.
} 
Berger y Luckmann (2015:172), la socialización secundaria es la internalización de "submundos" institucionales o basados en instituciones. El alcance de estos submundos está determinado a partir de la complejidad en la división del trabajo y la distribución del conocimiento.

Así, un grupo de referencia, según Merton (2002), va a ser aquel en el que el individuo se siente relacionado por sus normas, valores, emociones y circunstancias, es decir, cada miembro del grupo se siente integrado e identificado a la estructura social del grupo.

Existen diversas formas en las que se puede observar la socialización secundaria de los individuos. Por ejemplo: la escuela, grupos musicales, deportivos, culturales, grupos en los que se encuentra a los pares, aquellos con los que se tiene una relación de afinidad con algún elemento social, es decir: el gusto por algún instrumento musical, algún deporte, consumos culturales, etc. La banda o la pandilla resulta ser un agente socializador de conductas desviadas. En el actual contexto contemporáneo, donde la individualidad predomina en las situaciones sociales, estas agrupaciones, que no han dejado de existir, se han resignificado.

Para Kessler (2004:65), las bandas son una forma de protección entre los jóvenes que viven en contextos de violencia. Sánchez León (citado por Kessler, 2004), en su estudio sobre la vida delictiva de Lima, Perú, considera que existe una diferencia entre pandilla y banda. Al respecto, mientras la pandilla combina actividades propias de la edad de los jóvenes con los delitos, la banda se dedica exclusivamente a la actividad delictiva. En este sentido, el delito no es la razón o el motivo de agrupamiento, más bien es parte de las actividades del grupo que dan organización a un contexto caracterizado por el desorden.

En la situación de grupo, según Matza (2014), los jóvenes delincuentes se asumen como adherentes a una subcultura delictiva, como un miembro comprometido con una perspectiva contestataria a la que justifica. Ese compromiso termina en el momento en que se ha conseguido el objetivo o el grupo ya no sea de utilidad para conseguir los fines que el sujeto adherente se ha propuesto.

\section{Conclusiones}

En este reporte preliminar de investigación, estudiamos a los jóvenes que han vivido un proceso carcelario en los Centros de Internamiento para Adolescentes de Pachuca, Hidalgo (México). Nuestro objetivo es analizar la trayectoria de vida de estos jóvenes, tratando de identificar en sus procesos de socialización las causas de sus conductas desviadas.
Partimos teóricamente de la sociología de la desviación, que considera que la conducta desviada es criminalizada y etiquetados como delincuentes los que la mantienen, pues se consideran que quebrantan un orden social determinado socio-histórica y contextualmente a través de intercambios que definen la naturaleza de las relaciones sociales y el tipo de vínculos afectivos que les acompañan.

Consideramos que los jóvenes delincuentes son conscientes de que sus actos van en contra de la ley y afectan a la sociedad donde viven, pero que internalizan sus conductas mediante técnicas de neutralización que les permiten negarlas o minimizar el daño que causan.

En cuanto al nacimiento de las trayectorias desviadas, de los jóvenes analizados, siguiendo la socialización de los mismos, identificamos los siguientes tipos: la familia utiliza la delincuencia como modus vivendi y la familia es permisiva con pequeños actos delictivos (socialización primaria); amigos, conocidos o compañeros que realizan pequeños actos delictivos y a los que se une el joven, oportunidad de delinquir, unirse a bandas o pandillas que delinquen (socialización secundaria).

En lo que sigue, es necesario indagar más, a través de las técnicas etnográficas que estamos utilizando (entrevistas en profundidad, observación, relatos de vida), sobre las trayectorias desviadas de los jóvenes que han delinquido. Ello a fin de encontrar las causas que los orillan a delinquir, y respuestas sobre qué podemos hacer como sociedad para reinsertarlos.

\section{References}

Azaola, Elena, 1990. La institución correccional en México.Una mirada extraviada. México D.F.: Siglo XXI.

Becker, Howard, 2009. Outsiders. Hacia una sociología de la desviación. México D.F.: Siglo XXI.

Berger, Peter \& Luckmann, Thomas, 2015. La construcción social de la realidad. Buenos Aires: Amorrortu.

Blumer, Herbert, 1981. El interaccionismo simbólico: perspectiva y método. Barcelona: Hora D.L..

Downes, David \& Rock, Paul, 2011. Sociología de la desviación. Barcelona: Gedisa.

Garland, David, 2012. La cultura del control. Crimen y orden social en la sociedad contemporánea. Barcelona: Gedisa.

Goffman, Erving, 2007. Internados: ensayos sobre la situación social de los enfermos mentales. Buenos Aires: Amorrortu.

Kessler, Gabriel, 2004. Sociología del delito amateur. México D.F.: Paidós. 
Matza, David, 2014. Delincuencia y deriva. Cómo y por qué algunos jóvenes llegan a quebrantar la ley. México D.F.: Siglo XXI.

Merton, Robert, 2002. Teoría y estructuras sociales. México D.F.: Fondo de Cultura Económica.

Reguillo, Rossana., 2010. Los jóvenes en México. México D.F.: Fondo de Cultura Económica.

Saraví, Gonzalo, 2015. Juventudes fragmentadas. Socialización, clase y cultura en la construcción de la desigualdad. México D.F.: FLACSOCIESAS.

Sutherland, Edwin, 1983. Ladrones profesionales. Madrid: La Piqueta.

Taguenca, Juan Antonio, 2016. La identidad de los jóvenes en los tiempos de la globalización. Revista Mexicana de Sociología, 78(4), pp. 633654 .

Taylor, Steven \& Bogdan, Robert, 1994. Introducción a los métodos cualitativos de investigación. La búsqueda de los significados. México D.F.: Paidós.

Valenzuela Arce, José Manuel; Nanteras, Alfredo \& Reguillo, Rossana, 2007. Las maras. Identidades júveniles al límite. México D.F. Universidad Autónoma Metropolitana-El Colegio de la Frontera Norte.

Wacquant, Loïc, 2010. Las dos caras de un gueto: ensayos sobre marginalización y penalización. México D.F.: Siglo XXI.

Wacquant, Loïc, 2012. Castigar a los pobres. El gobierno neoliberal de la inseguridad social. Barcelona: Gedisa. 